$\mathrm{J}$ o u r n a l of

Mathematics

and Applications

JMA No 38, pp 59-70 (2015)

\title{
On a class of meromorphic functions defined by the convolution
}

\author{
Jacek Dziok, Janusz Sokół, Jan Stankiewicz
}

ABSTRACT: In the present paper we define some classes of meromorphic functions with fixed argument of coefficients. Next we obtain coefficient estimates, distortion theorems, integral means inequalities, the radii of convexity and starlikeness and convolution properties for the defined class of functions.

AMS Subject Classification: Primary 30C45, secondary 30C80

Keywords and Phrases: meromorphic functions, fixed argument, subordination, convolution

\section{Dedicated to Professor Leon Mikołajczyk}

\section{Introduction}

Let $\widetilde{\mathcal{M}}$ denote the class of functions which are analytic in $\mathcal{D}=\mathcal{D}(1)$, where

$$
\mathcal{D}(r)=\{z \in \mathbb{C}: 0<|z|<r\} \quad(r \in(0,1])
$$

and let $\mathcal{M}^{k}\left(k \in \mathbb{N}_{0}:=\{0,1,2, \ldots\}\right)$ denote the class of functions $f \in \widetilde{\mathcal{M}}$ of the form

$$
f(z)=\frac{1}{z}+\sum_{n=k}^{\infty} a_{n} z^{n} \quad(z \in \mathcal{D}) .
$$

Moreover, let $\mathcal{M}:=\mathcal{M}^{0}$. Also, by $\mathcal{T}_{\theta} \quad(\theta \in \mathbb{R})$ we denote the class of functions $f \in \mathcal{M}$ of the form

$$
f(z)=\frac{1}{z}+e^{i \theta} \sum_{n=0}^{\infty}\left|a_{n}\right| z^{n} \quad(z \in \mathcal{D}) .
$$

The class $\mathcal{T}_{\theta}$ is called the class of meromorphic functions with fixed argument of coefficients. For $\theta=\pi$ we obtain the class $\mathcal{T}_{\pi}$ of meromorphic functions with negative

COPYRIGHT (C) by Publishing Department Rzeszów University of Technology P.O. Box 85, 35-959 Rzeszów, Poland 
coefficients. Classes of functions with fixed argument of coefficients were considered in [1, 2, 3, 4].

A function $f \in \mathcal{M}$ is said to be convex in $\mathcal{D}(r)$ if

$$
\mathfrak{R e}\left(1+\frac{z f^{\prime \prime}(z)}{f^{\prime}(z)}\right)<0(z \in \mathcal{D}(r)) .
$$

A function $f \in \mathcal{M}$ is said to be starlike in $\mathcal{D}(r)$ if

$$
\mathfrak{R e} \frac{z f^{\prime}(z)}{f(z)}<0 \quad(z \in \mathcal{D}(r)) .
$$

Let $\mathcal{B}$ be a subclass of the class $\mathcal{M}$. We define the radius of starlikeness of order $\alpha$ and the radius of convexity of order $\alpha$ for the class $\mathcal{B}$ by

$$
\begin{aligned}
& R_{\alpha}^{*}(\mathcal{B})=\inf _{f \in \mathcal{B}}\{\sup \{r \in(0,1]: f \text { is starlike in } \mathcal{D}(r)\}\} \\
& R_{\alpha}^{c}(\mathcal{B})=\inf _{f \in \mathcal{B}}\{\sup \{r \in(0,1]: f \text { is convex in } \mathcal{D}(r)\}\}
\end{aligned}
$$

respectively.

Let functions $f, F$ be analytic in $\mathcal{U}:=\mathcal{D} \cup\{0\}$. We say that $f$ is subordinate to $F$, and write $f(z) \prec F(z)$ (or simply $f \prec F$ ), if and only if there exists a function $\omega$ analytic in $\mathcal{U},|\omega(z)| \leq|z| \quad(z \in \mathcal{U})$, such that

$$
f(z)=F(\omega(z)) \quad(z \in \mathcal{U}) .
$$

In particular, if $F$ is univalent in $\mathcal{U}$, we have the following equivalence:

$$
f(z) \prec F(z) \Longleftrightarrow f(0)=F(0) \text { and } f(\mathcal{U}) \subset F(\mathcal{U}) .
$$

For functions $f, g \in \widetilde{\mathcal{M}}$ of the form

$$
f(z)=\sum_{n=-1}^{\infty} a_{n} z^{n} \text { and } g(z)=\sum_{n=-1}^{\infty} b_{n} z^{n},
$$

by $f * g$ we denote the Hadamard product (or convolution) of $f$ and $g$, defined by

$$
(f * g)(z)=\sum_{n=-1}^{\infty} a_{n} b_{n} z^{n} \quad(z \in \mathcal{D}) .
$$

Let $\varphi \in \mathcal{M}^{k}$ be a given function of the form

$$
\varphi(z)=\frac{1}{z}+\sum_{n=k}^{\infty} \alpha_{n} z^{n} \quad\left(z \in \mathcal{D} ; \alpha_{n}>0, n=k, k+1, \ldots\right) .
$$

Assume that $A, B$ are real parameters, $-1 \leq A<B \leq 1, \quad(\cos \theta<0$ or $B \neq 1)$. By $\mathcal{M}^{k}(\varphi ; A, B)$ we denote the class of functions $f \in \mathcal{M}^{k}$ such that

$$
z(\varphi * f)(z) \prec \frac{1+A z}{1+B z} .
$$


Now, we define the classes of functions with fixed argument of coefficients related to the class $\mathcal{M}^{k}(\varphi ; A, B)$. Let us denote

$$
\mathcal{M}_{\theta}^{k}(\varphi ; A, B):=\mathcal{T}_{\theta} \cap \mathcal{M}^{k}(\varphi ; A, B), \mathcal{M}(\varphi ; A, B):=\mathcal{M}^{0}(\varphi ; A, B) .
$$

In the present paper we obtain coefficient estimates, distortion theorems, integral means inequalities, and the radii of convexity and starlikeness for the class $\mathcal{M}_{\theta}^{k}(\varphi ; A, B)$. We also derive convolution properties for the class of functions.

\section{Coefficient estimates}

Before stating and proving coefficient estimates in the class $\mathcal{M}(\varphi ; A, B)$ we need the following lemma.

Lemma 1 [G] Let $f$ be a function of the form

$$
f(z)=\sum_{n=0}^{\infty} a_{n} z^{n}
$$

which is analytic in $\mathcal{D}$. If $f \prec g$ and $g$ is convex univalent in $\mathcal{U}$, then

$$
\left|a_{n}\right| \leq 1 \quad(n \in \mathbb{N}) .
$$

Theorem 1 If a function $f$ of the form (1) belongs to the class $\mathcal{M}(\varphi ; A, B)$, then

$$
\left|a_{n}\right| \leq \frac{B-A}{\alpha_{n}} \quad(n=0,1, \ldots),
$$

The result is sharp.

Proof. Let a function $f$ of the form (1) belong to the class $\mathcal{M}(\varphi ; A, B)$ and let us put

$$
g(z)=\frac{z(\varphi * f)(z)-1}{A-B} \quad \text { and } \quad h(z)=\frac{z}{1+B z} .
$$

Then, by (5), we have $g \prec h$. Since the function $g$ is given by

$$
g(z)=\sum_{n=0}^{\infty} \frac{\alpha_{n}}{A-B} a_{n} z^{n+1}
$$

and the function $h$ is convex univalent in $\mathcal{U}$, by Lemma 1 we obtain

$$
\frac{\alpha_{n}}{B-A}\left|a_{n}\right| \leq 1 \quad\left(n \in \mathbb{N}_{0}\right)
$$

Thus we have (6). The Equality in (7) holds for the functions $g_{n}$ of the form

$$
g_{n}(z)=h\left(z^{n+1}\right)=z^{n+1}+\sum_{j=n+2}^{\infty} b_{j} z^{j} \quad(n=0,1, \ldots),
$$


for some $b_{j}(j=n+2, n+3, \ldots)$. Consequently, the equality in $(6)$ holds true for the functions $f_{n}$ of the form

$$
f_{n}(z)=\frac{1}{z}+\frac{A-B}{\alpha_{n}} z^{n}+\sum_{j=n+1}^{\infty} \frac{A-B}{\alpha_{j}} b_{j+1} z^{j} \quad(n=0,1, \ldots) .
$$

Theorem 2 If a function $f$ of the form (2) belongs to the class $\mathcal{M}_{\theta}^{k}(\varphi ; A, B)$, then

$$
\sum_{n=k}^{\infty} \alpha_{n}\left|a_{n}\right| \leq \delta(\theta ; A, B)
$$

where

$$
\delta(\theta ; A, B):=\frac{B-A}{\sqrt{1-B^{2} \sin ^{2} \theta}-B \cos \theta} .
$$

Proof. Let a function $f$ belong to the class $\mathcal{M}_{\theta}^{k}(\varphi ; A, B)$. Then, by (5) and the definition of subordination, we have

$$
z(\varphi * f)(z)=\frac{1+A \omega(z)}{1+B \omega(z)}
$$

where $\omega(0)=0$ and $|\omega(z)|<1$ for $z \in \mathcal{U}$. Thus we obtain

$$
|z(\varphi * f)(z)-1|<|B z(\varphi * f)(z)-A| \quad(z \in \mathcal{D}) .
$$

Hence, by (2), we have

$$
\left|\sum_{n=k}^{\infty} \alpha_{n}\right| a_{n}\left|z^{n+1}\right|<\left|B-A+B e^{i \theta} \sum_{n=k}^{\infty} \alpha_{n}\right| a_{n}\left|z^{n+1}\right| \quad(z \in \mathcal{D}) .
$$

Putting $z=r(0 \leq r<1)$, we find that

$$
|w|<\left|B-A+B w e^{i \theta}\right|,
$$

where, for convenience,

$$
w=\sum_{n=k}^{\infty} \alpha_{n}\left|a_{n}\right| r^{n+1} .
$$

Since $w$ is a real number, by (11) we have

$$
\left(1-B^{2}\right) w^{2}-[2 B(B-A) \cos \theta] w-(B-A)^{2}<0 .
$$

Solving this inequality with respect to $w$, we obtain

$$
\sum_{n=k}^{\infty} \alpha_{n}\left|a_{n}\right| r^{n+1}<\delta(\theta ; A, B)
$$

which, upon letting $r \rightarrow 1^{-}$, readily yields the assertion (8) of Theorem 1 . 
On a class of meromorphic functions defined by the convolution

Theorem 3 A function $f$ of the form (2) belongs to the class $\mathcal{M}_{\pi}^{k}(\varphi ; A, B)$ if and only if

$$
\sum_{n=k}^{\infty} \alpha_{n}\left|a_{n}\right| \leq \frac{B-A}{1+B}
$$

Proof. By virtue of Theorem 1, we only need to show that the condition 12 is the sufficient condition. Let a function $f$ of the form (2) satisfy the condition (12). Then, in view of 10 , it is sufficient to prove that

$$
\left|\sum_{n=k}^{\infty} \alpha_{n}\right| a_{n}\left|z^{n+1}\right|-\left|B-A-B \sum_{n=k}^{\infty} \alpha_{n}\right| a_{n}\left|z^{n+1}\right|<0 \quad(z \in \mathcal{D}) .
$$

Indeed, letting $|z|=r(0<r<1)$, we have

$$
\begin{aligned}
& \left|\sum_{n=k}^{\infty} \alpha_{n}\right| a_{n}\left|z^{n+1}\right|-\left|B-A-B \sum_{n=k}^{\infty} \alpha_{n}\right| a_{n}\left|z^{n+1}\right| \\
& \leq\left(\sum_{n=k}^{\infty} \alpha_{n}\left|a_{n}\right| r^{n+1}\right)-\left(B-A-B \sum_{n=k}^{\infty} \alpha_{n}\left|a_{n}\right| r^{n+1}\right) \\
& <(1+B) \sum_{n=k}^{\infty} \alpha_{n}\left|a_{n}\right|-(B-A) \leq 0,
\end{aligned}
$$

which implies that $f \in \mathcal{M}_{\pi}^{k}(\varphi ; A, B)$.

Theorem 2 readily yields

Corollary 1 If a function $f$ of the form (2) belongs to the class $\mathcal{M}_{\theta}^{k}(\varphi ; A, B)$, then

$$
\left|a_{n}\right| \leq \frac{\delta(\theta ; A, B)}{\alpha_{n}} \quad(n=k, k+1, \ldots)
$$

where $\delta(\theta ; A, B)$ is defined by (9). The result is sharp for $\theta=\pi$. Then the functions $f_{n}$ of the form

$$
f_{n}(z)=\frac{1}{z}-\frac{B-A}{(1+B) \alpha_{n}} z^{n} \quad(z \in \mathcal{D} ; n=k, k+1, \ldots)
$$

are the extremal functions.

\section{Distortion theorems}

From Theorem 2 we have the following lemma.

Lemma 2 Let a function $f$ of the form (2) belong to the class $\mathcal{M}_{\theta}^{k}(\varphi ; A, B)$. If the sequence $\left\{\alpha_{n}\right\}$ defined by (4) satisfies the inequality

$$
\alpha_{k} \leq \alpha_{n} \quad(n=k, k+1, \ldots)
$$


then

$$
\sum_{n=k}^{\infty}\left|a_{n}\right| \leq \frac{\delta(\theta ; A, B)}{\alpha_{k}}
$$

Moreover, if

$$
n \alpha_{k} \leq \alpha_{n} \quad(k \geq 1, n=k, k+1, \ldots)
$$

then

$$
\sum_{n=k}^{\infty} n\left|a_{n}\right| \leq \frac{k \delta(\theta ; A, B)}{\alpha_{k}}
$$

Theorem 4 Let a function $f$ belong to the class $\mathcal{M}_{\theta}^{k}(\varphi ; A, B)$. If the sequence $\left\{\alpha_{n}\right\}$ defined by (4) satisfies (15), then

$$
\frac{1}{r}-\frac{\delta(\theta ; A, B)}{\alpha_{k}} r^{k} \leq|f(z)| \leq \frac{1}{r}+\frac{\delta(\theta ; A, B)}{\alpha_{k}} r^{k} \quad(|z|=r<1) .
$$

Moreover, if 16 holds, then

$$
\frac{1}{r^{2}}-\frac{k \delta(\theta ; A, B)}{\alpha_{k}} r^{k-1} \leq\left|f^{\prime}(z)\right| \leq \frac{1}{r^{2}}+\frac{k \delta(\theta ; A, B)}{\alpha_{k}} r^{k-1} \quad(|z|=r<1) .
$$

The result is sharp for $\theta=\pi$, with the extremal function $f_{k}$ of the form (14).

Proof. Let a function $f$ of the form (2) belong to the class $\mathcal{M}_{\theta}^{k}(\varphi ; A, B),|z|=r<$ 1. Since

$$
|f(z)|=\left|\frac{1}{z}+e^{i \theta} \sum_{n=k}^{\infty} a_{n} z^{n}\right| \leq \frac{1}{r}+\sum_{n=k}^{\infty}\left|a_{n}\right| r^{n} \leq \frac{1}{r}+\sum_{n=k}^{\infty}\left|a_{n}\right|
$$

and

$$
|f(z)|=\left|\frac{1}{z}+e^{i \theta} \sum_{n=k}^{\infty} a_{n} z^{n}\right| \geq \frac{1}{r}-\sum_{n=k}^{\infty}\left|a_{n}\right| r^{n} \geq \frac{1}{r}-\sum_{n=k}^{\infty}\left|a_{n}\right|,
$$

then by Lemma 2 we have (17). Analogously we prove (18).

\section{$4 \quad$ Integral means inequalities}

Due to Littlewood [7] we obtain integral means inequalities for the functions from the class $\mathcal{M}_{\theta}^{k}(\varphi ; A, B)$.

Lemma 3 [7]. Let function $f, g$ be analytic in $\mathcal{U}$. If $f \prec g$, then

$$
\int_{0}^{2 \pi}\left|f\left(r e^{i t}\right)\right|^{\lambda} \mathrm{d} t \leq \int_{0}^{2 \pi}\left|g\left(r e^{i t}\right)\right|^{\lambda} \mathrm{d} t \quad(0<r<1, \lambda>0) .
$$


Silverman [8] found that the function

$$
g(z)=z-\frac{z^{2}}{2} \quad(z \in \mathcal{D}),
$$

is often extremal over the family of functions with negative coefficients. He applied this function to resolve integral means inequality, conjectured in [9] and settled in [10, that 19] holds true for all functions $f$ with negative coefficients. In [10] he also proved his conjecture for some subclasses of $\mathcal{T}_{\pi}$.

Applying Lemma 3 and Theorem 2 we prove the following result.

Theorem 5 Let the sequence $\left\{\alpha_{n}\right\}$ defined by (4) satisfy the inequality (15). If $f \in$ $\mathcal{M}_{\theta}^{0}(\varphi ; A, B)$, then

$$
\int_{0}^{2 \pi}\left|f\left(r e^{i t}\right)\right|^{\lambda} \mathrm{d} t \leq \int_{0}^{2 \pi}\left|g\left(r e^{i t}\right)\right|^{\lambda} \mathrm{d} t \quad(0<r<1, \lambda>0),
$$

where

$$
g(z)=\frac{1}{z}+e^{i \theta} \frac{\delta(\theta ; A, B)}{\alpha_{0}} \quad(z \in \mathcal{D}) .
$$

Proof. For function $f$ of the form (2), the inequality $(20)$ is equivalent to the following:

$$
\int_{0}^{2 \pi}\left|1+e^{i \theta} \sum_{n=0}^{\infty}\right| a_{n}\left|z^{n+1}\right|^{\lambda} \mathrm{d} t \leq \int_{0}^{2 \pi}\left|1+e^{i \theta} \frac{\delta(\theta ; A, B)}{\alpha_{0}} z\right|^{\lambda} \mathrm{d} t .
$$

By Lemma 3, it suffices to show that

$$
\sum_{n=0}^{\infty}\left|a_{n}\right| z^{n+1} \prec \frac{\delta(\theta ; A, B)}{\alpha_{0}} z .
$$

Setting

$$
w(z)=\sum_{n=0}^{\infty} \frac{\alpha_{0}}{\delta(\theta ; A, B)} a_{n} z^{n+1} \quad(z \in \mathcal{D})
$$

and using (15) and Theorem 2 we obtain

$$
|w(z)|=\left|\sum_{n=0}^{\infty} \frac{\alpha_{0}}{\delta(\theta ; A, B)} a_{n} z^{n+1}\right| \leq|z| \sum_{n=0}^{\infty} \frac{\alpha_{n}}{\delta(\theta ; A, B)}\left|a_{n}\right| \leq|z| \quad(z \in \mathcal{D}) .
$$

Since

$$
\sum_{n=0}^{\infty} a_{n} z^{n+1}=\frac{\delta(\theta ; A, B)}{\alpha_{0}} w(z) \quad(z \in \mathcal{D}),
$$

by definition od subordination we have 21 and this completes the proof. 


\section{The radii of convexity and starlikeness}

Theorem 6 If a function $f$ belongs to the class $\mathcal{M}_{\theta}^{k}(\varphi ; A, B), k \geq 1$, then $f$ is starlike in the disk $\mathcal{D}\left(r^{*}\right)$, where

$$
r^{*}:=\inf _{n \geq k}\left(\frac{\alpha_{n}}{n \delta(\theta, A, B)}\right)^{\frac{1}{n+1}}
$$

and $\delta(\theta, A, B),\left\{\alpha_{n}\right\}$ are defined by (9) and (4), respectively. For $\theta=\pi$, the result is sharp, that is

$$
R^{*}\left(\mathcal{M}_{\pi}^{k}(\varphi ; A, B)\right)=r^{*}
$$

Proof. A function $f \in \mathcal{M}^{k}$ of the form (2) is starlike in the disk $\mathcal{D}(r)$ if and only if it satisfies the condition $(3)$ or if

$$
\left|\frac{z f^{\prime}(z)+f(z)}{z f^{\prime}(z)-f(z)}\right|<1 \quad(z \in \mathcal{D}(r)) .
$$

Since

$$
\left|\frac{z f^{\prime}(z)+f(z)}{z f^{\prime}(z)-f(z)}\right|=\left|\frac{e^{i \theta} \sum_{n=k}^{\infty}(n+1)\left|a_{n}\right| z^{n}}{\frac{2}{z}-e^{i \theta} \sum_{n=k}^{\infty}(n-1)\left|a_{n}\right| z^{n}}\right| \leq \frac{\sum_{n=k}^{\infty}(n+1)\left|a_{n}\right||z|^{n+1}}{2-\sum_{n=k}^{\infty}(n-1)\left|a_{n}\right||z|^{n+1}},
$$

putting $|z|=r$ the condition 23 be true if

$$
\sum_{n=k}^{\infty} n\left|a_{n}\right| r^{n+1} \leq 1
$$

By Theorem 2, we have

$$
\sum_{n=k}^{\infty} \frac{\alpha_{n}}{\delta(\theta, A, B)}\left|a_{n}\right| \leq 1,
$$

Thus, the condition (24) be true if

$$
n r^{n+1} \leq \frac{\alpha_{n}}{\delta(\theta, A, B)} \quad(n=k, k+1, \ldots),
$$

that is, if

$$
r \leq\left(\frac{\alpha_{n}}{n \delta(\theta, A, B)}\right)^{\frac{1}{n+1}} \quad(n=k, k+1, \ldots) .
$$

It follows that each function $f \in \mathcal{M}_{\theta}^{k}(\varphi ; A, B)$ is starlike in the disk $\mathcal{D}\left(r^{*}\right)$, where $r^{*}$ is defined by 22 . For $\theta=\pi$ the functions $f_{n}$ of the form 14 are extremal functions. 
Theorem 7 If a function $f$ belongs to the class $\mathcal{M}_{\theta}^{k}(\varphi ; A, B)$, then $f$ is convex in the disk $\mathcal{D}\left(r^{c}\right)$, where

$$
r^{c}:=\inf _{n \geq k}\left(\frac{\alpha_{n}}{n^{2} \delta(\theta, A, B)}\right)^{\frac{1}{n+1}}
$$

and $\delta(\theta, A, B),\left\{\alpha_{n}\right\}$ are defined by (9) and (4), respectively. For $\theta=\pi$, the result is sharp, that is,

$$
R^{c}\left(\mathcal{M}_{\pi}^{k}(\varphi ; A, B)\right)=r^{c}
$$

Proof. The proof is analogous to that of Theorem 4, and we omit the details.

\section{Cnonvolution properties}

Let

$$
f(z)=\frac{1}{z}+e^{i \alpha} \sum_{n=k}^{\infty}\left|a_{n}\right| z^{n}, \quad g(z)=\frac{1}{z}+e^{i \beta} \sum_{n=k}^{\infty}\left|b_{n}\right| z^{n} \quad(z \in \mathcal{D}) .
$$

We define modified Hadamard product for the functions $f, g$ as follows:

$$
f \circledast g(z)=\frac{1}{z}-\sum_{n=k}^{\infty}\left|a_{n}\right|\left|b_{n}\right| z^{n} \quad(z \in \mathcal{D}) .
$$

Theorem 8 Let $f \in \mathcal{M}_{\alpha}^{k}(\varphi ; A, B)$ and $g \in \mathcal{M}_{\beta}^{k}(\psi ; C, D)$. Then $f \circledast g \in$ $\mathcal{M}_{\pi}^{k}(\varphi * \psi ; E, F)$, whenever

$$
\delta(\pi, E, F) \geq \delta(\alpha, A, B) \delta(\beta, C, D)
$$

Proof. Let

$$
\psi(z)=\frac{1}{z}+\sum_{n=k}^{\infty} \beta_{n} z^{n} \quad\left(z \in \mathcal{D} ; \beta_{n}>0, n=k, k+1, \ldots\right)
$$

and let functions $f, g$ of the form 25 belong to the classes $\mathcal{M}_{\alpha}^{k}(\varphi ; A, B)$ and $\mathcal{M}_{\beta}^{k}(\psi ; C, D)$, respectively. From Theorem 2 we have

$$
\sum_{n=k}^{\infty} \frac{\alpha_{n}}{\delta(\alpha ; A, B)}\left|a_{n}\right| \leq 1, \quad \sum_{n=k}^{\infty} \frac{\beta_{n}}{\delta(\beta ; C, D)}\left|b_{n}\right| \leq 1
$$

Thus, by 26 we obtain

$$
\begin{aligned}
\sum_{n=k}^{\infty} \frac{\alpha_{n} \beta_{n}}{\delta(\pi, E, F)}\left|a_{n} b_{n}\right| & \leq \sum_{n=k}^{\infty} \frac{\alpha_{n} \beta_{n}}{\delta(\alpha ; A, B) \delta(\beta ; C, D)}\left|a_{n}\right|\left|b_{n}\right| \\
& \leq \sum_{n=k}^{\infty} \frac{\alpha_{n}}{\delta(\alpha ; A, B)}\left|a_{n}\right| \sum_{n=k}^{\infty} \frac{\beta_{n}}{\delta(\beta ; C, D)}\left|b_{n}\right| \leq 1
\end{aligned}
$$

Applying Theorem 3 we get $f \circledast g \in \mathcal{M}_{\pi}^{k}(\varphi * \psi ; E, F)$. 
Theorem 9 Let the sequence $\left\{\alpha_{n}\right\}$ defined by (4) satisfy the inequalities (15). If $f, g \in \mathcal{M}_{\theta}^{k}(\varphi ; A, B)$, then $f \circledast g \in \mathcal{M}_{\pi}^{k}(\varphi ; C, D)$, whenever

$$
(D-C) \alpha_{0} \geq(1+D)[\delta(\theta, A, B)]^{2} .
$$

Proof. Let a functions $f, g$ of the form 25 belong to the class $\mathcal{M}_{\alpha}^{k}(\varphi ; A, B)$. Then by Theorem 2 we have

$$
\sum_{n=k}^{\infty} \frac{\alpha_{n}}{\delta(\alpha ; A, B)}\left|a_{n}\right| \leq 1, \quad \sum_{n=k}^{\infty} \frac{\alpha_{n}}{\delta(\alpha ; A, B)}\left|b_{n}\right| \leq 1 .
$$

Thus, by the Cauchy-Schwarz inequality we obtain

$$
\sum_{n=k}^{\infty} \frac{\alpha_{n}}{\delta(\theta, A, B)} \sqrt{\left|a_{n} b_{n}\right|} \leq 1 .
$$

We have to prove that

$$
\sum_{k=2}^{\infty} \alpha_{n} \frac{1+D}{D-C}\left|a_{n} b_{n}\right| \leq 1
$$

Therefore, by 28 it is sufficient to show that

$$
\frac{1+D}{D-C}\left|a_{n} b_{n}\right| \leq \frac{1}{\delta(\theta, A, B)} \sqrt{\left|a_{n} b_{n}\right|} \quad(n \geq 2)
$$

or equivalently

$$
\sqrt{\left|a_{n} b_{n}\right|} \leq \frac{D-C}{(1+D) \delta(\theta, A, B)} \quad(n \geq 2) .
$$

From (28) we have

$$
\sqrt{\left|a_{n} b_{n}\right|} \leq \frac{\delta(\theta, A, B)}{\alpha_{n}} \quad(n \geq 2) .
$$

Consequently, we need only to prove that

$$
\frac{D-C}{(1+D) \delta(\theta, A, B)} \geq \frac{\delta(\theta, A, B)}{\alpha_{n}} \quad(n \geq 2)
$$

and this inequality follows from (27) and (15).

We note that for functions $f \in \mathcal{M}_{\alpha}^{k}(\varphi ; A, B)$ and $g \in \mathcal{M}_{\pi-\alpha}^{k}(\psi ; C, D)$ we have $f * g=f \circledast g$. Thus from Theorem 8 obtain following corollary.

Corollary 2 If $f \in \mathcal{M}_{\alpha}^{k}(\varphi ; A, B)$ and $g \in \mathcal{M}_{\pi-\alpha}^{k}(\psi ; C, D)$, then $f * g \in$ $\mathcal{M}_{\pi}^{k}(\varphi * \psi ; E, F)$, whenever

$$
\delta(\pi, E, F) \geq \delta(\alpha, A, B) \delta(\pi-\alpha, C, D) .
$$

Putting $\theta=\pi$ in Theorem 9 we obtain following corollary. 
Corollary 3 Let the sequence $\left\{\alpha_{n}\right\}$ defined by (4) satisfy (15). If $f, g \in \mathcal{M}_{\pi}^{k}(\varphi ; A, B)$, then $f \circledast g \in \mathcal{M}_{\pi}^{k}(\varphi ; C, D)$, whenever

$$
(D-C)(1+B)^{2} \alpha_{0} \geq(1+D)(B-A)^{2} .
$$

Putting $C=A$ and $D=B$ in Corollary 3 we obtain following corollary.

Corollary 4 Let the sequence $\left\{\alpha_{n}\right\}$ defined by (4) satisfy (15). If $f, g \in \mathcal{M}_{\pi}^{k}(\varphi ; A, B)$, then $f \circledast g \in \mathcal{M}_{\pi}^{k}(\varphi ; A, B)$, whenever

$$
\alpha_{0} \geq \frac{B-A}{1+B}
$$

Since for $\alpha=\beta=\pi, E=A$ and $F=B$ the condition 26) is true, then from Theorem 8 we have following corollary.

Corollary 5 If $f \in \mathcal{M}_{\pi}^{k}(\varphi ; A, B)$ and $g \in \mathcal{M}_{\pi}^{k}(\psi ; C, D)$, then

$$
f \circledast g \in \mathcal{M}_{\pi}^{k}(\varphi * \psi ; A, B) \cap \mathcal{M}_{\pi}^{k}(\varphi * \psi ; C, D) .
$$

\section{References}

[1] J. Dziok, Classes of functions defined by certain differential-integral operators, J. Comput. Appl. Math. 105(1999), 245-255.

[2] J. Dziok, Classes of $p$-valent analytic functions with fixed argument of coefficients, Demonstratio Math. 33(2000), 55-63.

[3] J. Dziok , H. M. Srivastava, Some subclasses of analytic functions with fixed argument of coefficients associated with the generalized hypergeometric function, Adv. Stud. Contemp. Math., 2002, 5:115-125.

[4] J. Dziok, J. Stankiewicz, On a class of $p$-valent analytic functions with fixed argument of coefficients defined by fractional calculus, Folia Scient. Univ. Tech. Resov. 21(1997), $19-35$.

[5] J. Stankiewicz. J. Waniurski, Some classes of univalent functions subordinate to linear transformation and their applications, Ann. Univ. Mariae Curie-Skłodowska, 1974, 9:85-94.

[6] W. Rogosiński, On the coefficients of subordinate functions, Proc. Lond. Math. Soc., 1943, 48:48-82.

[7] J. E. Littlewood, On inequalities in theory of functions, Proc. London Math. Soc., 1925, 23:481-519.

[8] H. Silverman, Univalent functions with negative coefficients, Proc. Amer. Math. Soc., 1975, 51:109-116. 
[9] H. Silverman, A survey with open problems on univalent functions whose coefficients are negative, Rocky Mountain J. Math., 1991, 21:1099-1125.

[10] H. Silverman, Integral means for univalent functions with negative coefficients, Houston J. Math., 1997, 23:169-174.

DOI: $10.7862 / \mathrm{rf.2015.5}$

J.Dziok - corresponding author

email: jdziok@ur.edu.pl

Faculty of Mathematics and Natural Sciences, University of Rzeszów, 35-310 Rzeszów, Poland

Janusz Sokół

email: jsokol@prz.edu.pl

Jan Stankiewicz

email: jstan@prz.edu.pl

Department of Mathematics,

Rzeszów University of Technology,

Al. Powstańców Warszawy 12,

35-959 Rzeszów, Poland

Received 20.10.2014 